ThEOREM 10. For the derivatives of functions $f_{p}(z)$ of class $S$ :

$$
1-|z| \leqq\left|f_{p}^{\prime}(z)\right| \leqq 1+|z| \text {. }
$$

These inequalities are sharp. They are attained by $f_{p}(z)=z-z^{2} / 2$ at $z= \pm r, r$ real.

TEMPLE UNIVERSITY

\title{
A REMARK ON REVERSIBLE MATRICES
}

\section{S. MACPHAIL}

The matrix $A=\left(a_{n k}\right)$ is called reversible if for each $y=\left\{y_{n}\right\} \in(c)$ the equations $y_{n}=\sum_{k=0}^{\infty} a_{n k} x_{k}(n=0,1, \cdots)$ have exactly one solution $x=\left\{x_{k}\right\}$. In this case there exist $[1 ; 4]$ constants $c_{k}, b_{k n}$ with $\sum_{n}\left|b_{k n}\right|<\infty$ for each $k$, such that

$$
x_{k}=c_{k} \lim _{n \rightarrow \infty} y_{n}+\sum_{n=0}^{\infty} b_{k n} y_{n} \quad(k=0,1, \cdots) .
$$

It is further stated in $[1$, p. 50$]$ that the $c_{k}$ are bounded. This is questioned in [4], where it is pointed out that if the $c_{k}$ were generally bounded they would have to be almost all zero, but this remark does not dispose of the matter, for it might conceivably be a true theorem that for each reversible matrix the $c_{k}$ are almost all zero. (For rowfinite matrices, all $c_{k}$ vanish; [3].) The example given in [4, p. 47] seems inconclusive. The purpose of this note is to show by a very simple example that in fact the $c_{k}$ need not be bounded.

Consider the transformation

$$
y_{2 m}=\sum_{p=0}^{m} x_{2 p}, \quad y_{2 m+1}=2^{-m} x_{2 m+1}+\sum_{p=0}^{\infty} x_{2 p},
$$

where $m=0,1, \cdots$. For each $y \in(c)$ we have

$$
x_{2 m+1}=2^{m}\left(y_{2 m+1}-\lim _{n} y_{n}\right)
$$

thus $c_{2 m+1}=-2^{m}$ is not bounded.

This has a bearing on a paper [2] in which the following theorem is stated:

Received by the editors June 22, 1953. 
Theorem. $A$ reversible conservative matrix $A$ sums a divergent sequence if and only if $\left\|A^{-1}\right\|=\infty$.

Here $\left\|A^{-1}\right\|$ is defined as $\sup _{k}\left(\left|c_{k}\right|+\sum_{n}\left|b_{k n}\right|\right)$ with $c_{k}, b_{k n}$ as above, and the theorem is proved under the assumption that the $c_{k}$ are bounded. There is, however, no difficulty in allowing for the possibility of the $c_{k}$ being unbounded. For then in the sufficiency part of the proof $[2, \mathrm{p} .915]$, we note that if $\|B\|=\sup _{k} \sum_{n}\left|b_{k n}\right|<\infty$, we may take $y=\{1,1,1, \ldots\}$, and obtain at once from (1) a divergent sequence $x$ which is $A$-summable, while if $\|B\|=\infty$, we can find by classical methods a sequence $y=\left\{y_{n}\right\}$ with $\lim _{n} y_{n}=0$ and $B y$ unbounded. For such a sequence the $c_{k}$ are immaterial and we have simply $x=B y$. This completes the proof.

\section{REFERENCES}

1. S. Banach, Théorie des operations linéaires, Warsaw, 1932.

2. A. Wilansky, $A$ necessary and sufficient condition that a summability method be stronger than convergence, Bull. Amer. Math. Soc. vol. 55 (1949) pp. 914-916.

3. - Convergence fields of row-finite and row-infinite reversible matrices. Proc. Amer. Math. Soc. vol. 3 (1952) pp. 389-391.

4. K. Zeller, FK-Räume und Matrixtransformationen, Math. Zeit. vol. 58 (1953) pp. $46-48$.

Carlegon College, Ottawa, Canada 